\title{
CONSTRUCTION OF HOMOMORPHISMS OF $M$-CONTINUOUS LATTICES
}

\author{
XIAO-QUAN XU
}

\begin{abstract}
We present a direct approach to constructing homomorphisms of $M$-continuous lattices, a generalization of continuous lattices, into the unit interval, and show that an $M$-continuous lattice has sufficiently many homomorphisms into the unit interval to separate the points.
\end{abstract}

In the past twenty years the concept of a continuous lattice and its generalizations have attracted more and more attention. It was the pioneering work of Dana Scott [15], [16] which led to the discovery that algebraic lattices and their generalization, continuous lattices, could be used to assign meanings to programs written in high-level programming languages. On the purely mathematical side, research into the structure theory of compact semilattices led Lawson [9] and others [7], [8] to consider the category of those compact semilattices which admit a basis of subsemilattice neighborhoods at each point. It was discovered in [8] that those objects are precisely the continuous lattices of Scott. One of the most important features of continuous lattices is that they admit sufficiently many homomorphisms (that is, mappings which preserve arbitrary infs and directed sups) into the unit interval to separate the points. The topological form of this result is due to Lawson [9].

For a complete lattice $L$ and a family $M$ of subsets of $L$, we define a corresponding relation $\ll_{M}$ on $L$ by $x \ll_{M} y$ if and only if, for each $S \in M$, $y \leq \bigvee S$ implies there exists $s \in S$ such that $x \leq s ; L$ is called $M$-continuous if the relation $\ll_{M}$ satisfies the interpolation property (i.e. for every $x, y \in$ $L, x \ll_{M} y$ implies there is $z \in L$ such that $\left.x \ll_{M} z \ll_{M} y\right)$ and $x=$ $\bigvee\left\{y \in L \mid y \ll_{M} x\right\}$ for all $x \in L$. For a complete lattice $T$, a map $f: L \rightarrow T$ is called an $M$-morphism, or, briefly, a homomorphism, if $f$ preserves arbitrary infs and $M$-sups.

This paper is mainly devoted to the construction of homomorphisms of $M$ continuous lattices into the unit interval $[0,1]$. We present a direct approach to the construction of such homomorphisms and show that $M$-continuous lattices admit enough homomorphisms into $[0,1]$ to separate points.

Received by the editors February 3, 1994 and, in revised form on July 21, 1994; originally communicated to the Proceedings of the AMS by Andreas R. Blass.

1991 Mathematics Subject Classification. Primary 06B15, 06B35, 06 D05.

Key words and phrases. $M$-continuity, $M$-distributivity, $M$-below relation, embedding.

This research was partially supported by the National Tianyuan Mathematics Foundation of China and the National Natural Science Foundation of China and the Natural Science Foundation of Jiangxi Province.

(C)1995 American Mathematical Society 
The general idea of constructing morphisms of the right kind into chains by using maximal complete strict chains relative to suitable auxiliary relations dates from the 1950s; forerunners are to be found in Raney's classical papers on completely distributive lattices [13], [14]. The classical technique of Raney and Bruns [5], based on the investigations of maximal complete strict chains of suitable auxiliary relations, is skillful and requires AC (the axiom of choice). Moreover, it cannot be applied to the case of posets. Comparatively, the technique presented in this paper is simpler and requires only the axiom of $\omega$ dependent choices; besides, it can be directly applied to the case of posets. In a forthcoming article we will use the technique to set up the embedding theory of posets.

\section{Preliminaries}

In this preliminary section we collect some well-known notation, definitions and results needed later on. The reader wishing more details can consult [1], [2], [7], and [11].

Let $P$ be a poset and let $x \in P$. We define $\downarrow x=\{y \in P \mid y \leq x\}$. The set $\downarrow S=\bigcup\{\downarrow x \mid x \in S\}$ is called the lower set generated by $S$. The family of all subsets of $P$ is denoted by $\mathscr{P}(P)$.

Denote by $\mathrm{INF}^{\uparrow}$ (resp., INF $\cap$ SUP) the category of all complete lattices and all maps preserving arbitrary infs and directed sups (resp., all complete lattice morphisms).

In what follows $L$ is always a complete lattice and $M$ is a subset system of $L$, i.e. $M \subset \mathscr{P}(L)$. Define $I_{M}(L)=\{\downarrow S \mid S \in M\}$. $I_{M}(L)$ (ordered by inclusion) is called the poset of all $M$-generated lower sets of $L$.

Important examples of subset systems of $L$ are

$\mathscr{P}(=\mathscr{P}(L))$, the family of all subsets of $L$,

$\mathscr{D}(=\mathscr{D}(L))$, the family of all directed subsets of $L$, and

$\mathscr{F}(=\mathscr{F}(L))$, the family of all finite subsets of $L$.

The following conditions on $M$ are relevant:

(•) for every $x \in L$ there exists $S \in M$ with $x=\bigvee S$,

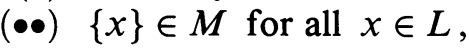

(†) for each family $\left\{S_{i} \mid i \in I\right\} \subset M$, there exists $S \in M$ such that

$$
S \subset \downarrow\left\{\bigwedge_{i \in I} \varphi(i) \mid \varphi \in \prod_{i} S_{i}\right\} \quad \text { and } \quad \bigvee S=\bigvee\left\{\bigwedge_{i \in I} \varphi(i) \mid \varphi \in \prod_{i} S_{i}\right\} \text {, }
$$

(††) for each family $\left\{S_{i} \mid i \in I\right\} \subset M$,

$$
\left\{\bigwedge_{i \in I} \varphi(i) \mid \varphi \in \prod_{i} S_{i}\right\} \in M
$$

(*) if $\left\{S_{i} \mid i \in I\right\} \subset M$ and $\left\{\bigvee S_{i} \mid i \in I\right\} \in M$, then there exists $S \in M$ such that

$$
S \subset \downarrow \bigcup_{i \in I} S_{i} \text { and } \bigvee S=\bigvee \bigcup_{i \in I} S_{i}
$$

(**) if $\left\{S_{i} \mid i \in I\right\} \subset M$ and $\left\{\bigvee S_{i} \mid i \in I\right\} \in M$, then $\bigcup_{i \in I} S_{i} \in M$.

G. Bruns [5] calls a subset system $M$ distributively closed whenever it satisfies $(\bullet \bullet),(\dagger \dagger)$, and $(* *)$. Unfortunately, $\mathscr{D}$ is in general not distributively closed $((* *)$ is the troublemaker). 
An important relation on $L$ depending on $M$ is the $M$-below relation $\ll_{M}: x \ll_{M} y$ if and only if for each $S \in M$, if $y \leq \bigvee S$, then there exists $s \in S$ such that $x \leq s$. For $x \in L$, let $\Downarrow_{M} x=\left\{y \in L \mid y \ll_{M} x\right\}$. Clearly, $\Downarrow_{M} x=\bigcap\left\{A \in I_{M}(L) \mid y \leq \bigvee A\right\}$. In the case $M=\mathscr{D}$, the relation $\ll_{M}$ is the well-known way below relation $\ll$.

1.1. Definition. A complete lattice $L$ is called a continuous lattice if $x=$ $\bigvee\{y \in L \mid y \ll x\}$ for all $x \in L$.

1.2. Definition. Let $M$ be a subset system of a complete lattice $L . L$ is called $M$-distributive if, for every family $\left\{S_{i} \mid i \in I\right\} \subset M$,

$$
\bigwedge\left\{\bigvee S_{i} \mid i \in I\right\}=\bigvee\left\{\bigwedge_{i \in I} \varphi(i) \mid \varphi \in \prod_{i} S_{i}\right\}
$$

$L$ is called completely distributive, if $L$ is $\mathscr{P}$-distributive.

1.3. Definition. A binary relation $\sqsubset$ on a nonempty set $X$ is said to have the interpolation property, if it satisfies

(INT) For all $x, y \in X$ with $x \sqsubset y$, there exists $z \in X$ such that $x \sqsubset z \sqsubset$ $y$.

1.4. Definition. A binary relation $\prec$ on a complete lattice $L$ is called approximating, if $x=\bigvee\{y \in L \mid y \prec x\}$ for all $x \in L$.

1.5. Definition. Let $L$ be a complete lattice and let $M$ be a subset system which satisfies $(\bullet)$.

(1) $L$ is called weakly $M$-continuous, if the relation $\ll_{M}$ on $L$ is approximating.

(2) $L$ is called $M$-continuous, if the relation $\ll_{M}$ on $L$ is approximating and satisfies (INT) .

(3) $L$ is called strongly $M$-continuous, if the relation $\ll_{M}$ on $L$ is approximating and satisfies (INT), and $\Downarrow_{M} x \in I_{M}(L)$ for all $x \in L$.

1.6. Remark. The concept of $M$-continuity in Definition 1.5 is different from the one in [2], [11]. Here it is not required that $\Downarrow_{M} x \in I_{M}(L)$ for all $x \in L$, but the relation $\ll_{M}$ is required to satisfy the celebrated interpolation property.

The following result is well known (see [1], [11]):

1.7. Lemma. Let $M$ be a subset system of a complete lattice $L$ and let $M$ satisfy $(\bullet)$. Then $L$ is weakly $M$-continuous if and only if $L$ is $M$-distributive.

1.8. Lemma (Bandelt [1]). Let $L$ be a complete lattice and let $M$ be a subset system which satisfies $(\bullet)$. If $L$ is weakly $M$-continuous, then

(1) $M$ satisfies ( $\dagger$ ) if and only if $\Downarrow_{M} x \in I_{M}(L)$ for all $x \in L$ (i.e. $L$ is $M$-continuous in the sense of [1], [11]);

(2) if $M$ satisfies ( $\dagger$ ), then the relation $\ll_{M}$ on $L$ satisfies (INT) (i.e. $L$ is $M$-continuous) if and only if $M$ satisfies (*).

1.9. Corollary. Let $L$ be a complete lattice and let $M$ be a subset system which satisfies $(\bullet)$. If $L$ is $M$-distributive, then the following conditions are equivalent:

(1) $L$ is strongly $M$-continuous;

(2) $M$ satisfies ( $\dagger)$ and $(*)$; 
(3) $M$ satisfies (*) and $\Downarrow_{M} x \in I_{M}(L)$ for each $x \in L$.

1.10. Definition. Let $S$ and $T$ be two posets. A pair $(f, g)$ of maps $f: S \rightarrow$ $T$ and $g: T \rightarrow S$ is called a Galois connection or an adjunction between $S$ and $T$ provided that

(1) both $f$ and $g$ are order preserving, and

(2) the relations $f(s) \geq t$ and $s \geq g(t)$ are equivalent for all pairs of elements $(s, t) \in S \times T$.

In an adjunction $(f, g)$, the map $f$ is called the upper adjoint and $g$ the lower adjoint.

1.11. Lemma. Let $f: S \rightarrow T$ and $g: T \rightarrow S$ be maps between posets. Consider the following conditions:

(1) $(f, g)$ is a Galois connection between $S$ and $T$,

(2) $f$ is order preserving and $g(t)=\min f^{-1}(\uparrow t)$ for each $t \in T$,

(3) $g$ is order preserving and $f(s)=\max g^{-1}(\downarrow s)$ for each $s \in S$, and

(4) $f$ preserves all existing infs and $g$ preserves all existing sups.

Then $(1) \Leftrightarrow(2) \Leftrightarrow(3) \Rightarrow(4)$. Consequently, in an adjunction one map uniquely determines the other.

\section{CONSTRUCTION OF $M$-MORPHISMS}

2.1. Definition. Let $M$ be a subset system of a complete lattice $L$ and let $T$ be a complete lattice. We say that a map $f: L \rightarrow T$ is an $M$-morphism, or, briefly, a homomorphism, if it preserves arbitrary infs and $M$-sups $(f$ is said to preserve $M$-sups, if $f(\bigvee S)=\bigvee f(S)$ for all $S \in M)$. The set of homomorphisms of $L$ into $T$ is denoted by $I M^{\uparrow}(L, T)$. For $f \in I M^{\uparrow}(L, T)$, if $f$ is injective, then $f$ is called an $I M^{\dagger}$-embedding of $L$ into $T$.

In the following definition the symbol $\omega$ denotes the ordinal number of the set of natural numbers.

2.2. Definition (The axiom of $\omega$ dependent choices- $-\mathrm{DC}_{\omega}$ ). Let $X$ be a set, let $u \in X$ and let $R$ be a binary relation on $X$. If for every $x \in X$ there is a $y \in X$ such that $x R y$, then there is a sequence $\left\{x_{n} \mid n<\omega\right\} \subset X$ such that $x_{0}=u$ and $x_{n} R x_{n+1}$ for all $n<\omega$.

$\mathrm{DC}_{\omega}$ was proposed by Bernays [4] in 1942 as a weaker version of the axiom of choice. We will use the symbol $\mathrm{ZFDC}_{\omega}$ to denote the theory obtained by adjoining $\mathrm{DC}_{\omega}$ to $\mathrm{ZF}$ (the Zermelo-Fraenkel set theory).

One can easily prove the following result.

2.3. Lemma $\left(\mathbf{Z F D C}_{\omega}\right)$. Let $\sqsubset$ be a binary relation on a set $X$ and let $B$ be the set of dyadic rational numbers in $[0,1]$. If $\sqsubset$ is transitive and satisfies (INT) (i.e. $\sqsubset$ is idempotent with respect to the relational product), then for $x, y \in X$ with $x \sqsubset y$, there is a family $\{x(b) \mid b \in B\} \subset X$ such that

(1) $x(0)=x, x(1)=y$, and

(2) $x\left(b_{1}\right) \sqsubset x\left(b_{2}\right)$ whenever $b_{1}<b_{2}$.

2.4. Remark. Let $X$ be a topological space and $o(X)$ be the complete lattice of all open sets of $X$. Define a relation $\sqsubset_{t}$ on $o(X)$ by $U \sqsubset_{t} V$ if and only if $\bar{U} \subset V$. Obviously. $\sqsubset_{t}$ is transitive. If $X$ is a normal space, then $\sqsubset_{t}$ satisfies (INT). For a pair $F, G$ of disjoint closed subsets of a normal space $X$, by 
normality of $X$, there is an open set $U$ such that $F \subset U \subset \bar{U} \subset X \backslash G$. The main technique used in the proof of Urysohn's lemma is to construct a family $\{U(b) \mid b \in B\} \subset o(X)$ such that

(1) $U(0)=U, U(1)=X \backslash G$, and

(2) $U\left(b_{1}\right) \sqsubset_{t} U\left(b_{2}\right)$ whenever $b_{1}<b_{2}$.

2.5. Lemma. Let $L$ be a complete lattice and let $M$ be a subset system which satisfies $(\bullet)$. Let the $M$-below relation $\ll_{M}$ satisfy $(\mathrm{INT})$. For each $(x, t) \in$ $L \times[0,1]$, define a map $M_{x . t}: L \rightarrow[0,1]$ by

$$
M_{x, t}(y)= \begin{cases}t, & \text { if } x \ll_{M} y, \\ 0, & \text { otherwise. }\end{cases}
$$

Then $M_{x, t}$ preserves $M$-sups.

Proof. Trivial.

Now, we proceed to the main results of this paper.

2.6. Theorem $\left(\mathbf{Z F D C}_{\omega}\right)$. Let $L$ be a complete lattice and let $M$ be a subset system which satisfies $(\bullet)$. Let the $M$-below relation $\ll_{M}$ satisfy (INT). Suppose that $x \leq y$ in $L$, Then the following statements are equivalent:

(1) There is a map $f \in I M^{\uparrow}(L,[0,1])$ with $f(x)=1$ and $f(y)=0$.

(2) There is $a u \in L$ with $u \ll_{M} x$ and $u \not y y$.

Proof. (1) $\Rightarrow(2)$. Let $g:[0,1] \rightarrow L$ be the lower adjoint of $f$ (i.e. $g(t)=$ $\bigwedge f^{-1}(\uparrow t)$ for each $\left.t \in[0,1]\right)$. Then $g\left(t_{1}\right) \ll_{M} g\left(t_{2}\right)$ whenever $t_{1}<t_{2}$. Let $u=g\left(\frac{1}{2}\right)$. Then $u \ll_{M} x$ and $u \not \leq y$.

$(2) \Rightarrow(1)$. Let $B$ be the set of dyadic rational numbers in $[0,1]$. Then by Lemma 2.3 , there is a family $\{x(b) \mid b \in B\} \subset L$ such that

(i) $x(0)=u, x(1)=x$, and

(ii) $x\left(b_{1}\right) \ll_{M} x\left(b_{2}\right)$ whenever $b_{1}<b_{2}$.

Let $f=\bigvee\left\{M_{x(b), b} \mid b \in B\right\}$, i.e. $f(z)=\bigvee\left\{b \in B \mid x(b) \ll_{M} z\right\}$ for each $z \in L$. Then $f(x)=1$ and $f(y)=0$. Define $d:[0,1] \rightarrow L$ by

$$
d(t)=\bigvee\{x(b) \mid b \in B \text { and } b<t\} .
$$

Then for all pairs of elements $(s, t) \in L \times[0,1], f(s) \geq t$ is equivalent to $s \geq d(t)$. It follows that $(f, d)$ is a Galois connection between $L$ and $[0,1]$. Therefore, by Lemmas 1.11 and 2.5 (both of them are true in $Z F$ ), $f \in I M^{\uparrow}(L,[0,1])$.

2.7. Corollary $\left(\mathbf{Z F D C}_{\omega}\right)$. Let $L$ be a complete lattice in which the way below relation « satisfies (INT). Suppose that $x \notin y$ in $L$. Then the following statements are equivalent:

(1) There is a map $f \in \operatorname{INF}^{\dagger}(L,[0,1])$ with $f(x)=1$ and $f(y)=0$.

(2) There is $a u \in L$ with $u \ll x$ and $u \not \leq y$.

Corollary 2.7 is an improvement of Proposition IV-2.20 of [7].

2.8. Corollary $\left(\mathbf{Z F D C}_{\omega}\right)$. Let $L$ be a complete lattice in which the totally below relation $\triangleleft(=\ll \mathscr{P})$ satisfies (INT). Suppose that $x \leq y$ in $L$. Then the following statements are equivalent:

(1) There is a map $f \in(\operatorname{INF} \cap \operatorname{SUP})(L,[0,1])$ with $f(x)=1$ and $f(y)=0$. 
(2) There is $a u \in L$ with $u \triangleleft x$ and $u \not \leq y$.

2.9. Theorem $\left(\mathbf{Z F D C}_{\omega}\right)$. Let $L$ be a complete lattice and let $M$ be a subset system which satisfies $(\bullet)$. Consider the following conditions:

(1) $L$ is $M$-continuous,

(2) $\operatorname{IM}^{\dagger}(L,[0,1])$ separates the points of $L$, and

(3) $L$ is weakly $M$-continuous.

Then $(1) \Rightarrow(2) \Rightarrow(3)$.

Proof. (1) $\Rightarrow$ (2) follows from Theorem 2.6.

(2) $\Rightarrow(3)$. For $x, y \in L$ with $x \leq y$, if $f(x) \leq f(y)$ for all $f \in$ $I M^{\dagger}(L,[0,1])$, then $f(x \wedge y)=f(x) \wedge f(y)=f(x)$ for all $f \in I M^{\uparrow}(L,[0,1])$. By (2) it follows that $x \leq y$. This contradiction shows that there is $f \in$ $I M^{\dagger}(L,[0,1])$ such that $f(y)<f(x)$. Select a $t \in[0,1]$ with $f(y)<t<$ $f(x)$. Let $g$ be the lower adjoint of $f$ and let $u=g(t)$. Then $u \ll_{M} x$ and $u \not y$. It follows that $x=\bigvee\left\{z \in L \mid z \ll_{M} x\right\}$ for all $x \in L$.

It is well known, in ZF, that $\Downarrow x \in I_{\mathscr{D}}(L)$ for each $x \in L$, and $L$ is continuous if and only if $L$ is $\mathscr{D}$-continuous (i.e. the way below relation $\ll$ on $L$ is approximating implies that $\ll$ satisfies (INT)). Thus we arrive at the following result.

2.10. Corollary $\left(\mathbf{Z F D C}_{\omega}\right)$. Let $L$ be a complete lattice. Then the following statements are equivalent:

(1) $L$ is strongly $\mathscr{D}$-continuous;

(2) $L$ is $\mathscr{D}$-continuous;

(3) $L$ is continuous;

(4) $\operatorname{INF}^{\dagger}(L,[0,1])$ separates the points of $L$.

2.11. Corollary $\left(\mathbf{Z F D C}_{\omega}\right)$. Let $L$ be a complete lattice. Then the following statements are equivalent:

(1) L is strongly $\mathscr{P}$-continuous;

(2) $L$ is $\mathscr{P}$-continuous;

(3) $L$ is weakly $\mathscr{P}$-continuous;

(4) $(\operatorname{INF} \cap \operatorname{SUP})(L,[0,1])$ separates the points of $L$.

\section{Applications}

In this section the axiom of choice is required.

By Lemma 1.7, Lemma 1.8, and Theorem 2.9, we obtain the following result.

3.1. Theorem. Let $L$ be a complete lattice and let $M$ be a subset system which satisfies $(\bullet)$. Consider the following conditions:

(1) L is strongly M-continuous,

(2) $L$ is $M$-continuous,

(3) $\operatorname{IM}^{\dagger}(L,[0,1])$ separates the points of $L$,

(4) There is an $I M^{\dagger}$-embedding of $L$ into a cube $[0,1]^{X}$,

(5) $L$ is weakly $M$-continuous, and

(6) $L$ is $M$-distributive.

Then the following implications and equivalences hold:

$$
(1) \Rightarrow(2) \Rightarrow(3) \Leftrightarrow(4) \Rightarrow(5) \Leftrightarrow(6) \text {. }
$$

If $M$ satisfies ( $\dagger)$ and $(*)$, then $(6) \Rightarrow(1)$ is true, and hence all six conditions are equivalent. 
3.2. Corollary (Lawson [7], [9]). Let L be a complete lattice. Then the following statements are equivalent:

(1) $L$ is continuous;

(2) $L$ is $\mathscr{D}$-distributive;

(3) $\operatorname{INF}^{\dagger}(L,[0,1])$ separates the points of $L$;

(4) There is an $\mathrm{INF}^{\dagger}$-embedding of $L$ into a cube $[0,1]^{X}$.

3.3. Corollary (Raney [13]; [14]). Let L be a complete lattice. Then the following statements are equivalent:

(1) The totally below relation $\triangleleft$ on $L$ is approximating,

(2) $L$ is completely distributive;

(3) $(\mathrm{INF} \cap \mathrm{SUP})(L,[0,1])$ separates the points of $L$;

(4) There is an INF $\cap$ SUP-embedding of $L$ into a cube $[0,1]^{X}$.

3.4. Corollary (Bruns [5]). Let $L$ be a complete lattice and let $M$ be a subset system which satisfies $(\bullet \bullet),(\dagger \dagger)$, and $(* *)$. Then the following statements are equivalent:

(1) The relation $\ll_{M}$ on $L$ is approximating;

(2) $L$ is $M$-distributive;

(3) $I M^{\dagger}(L,[0,1])$ separates the points of $L$;

(4) There is an $I M^{\uparrow}$-embedding of $L$ into a cube $[0,1]^{X}$.

3.5. Definition. Let $L$ be a complete lattice.

(1) $p \in L$ is called coprime, if the relation $p \leq x \vee y$ always implies $p \leq x$ or $p \leq y$. The set of coprime elements of $L$ is denoted by $\operatorname{COPRI}(L)$.

(2) A subset $B$ of $L$ is called a base of $L$, if $x=\bigvee\{b \in B \mid b \leq x\}$ for all $x \in L$.

(3) A subset $S$ of $L$ is called a $\Lambda$-sublattice of $L$, if the inclusion map $S \rightarrow L$ is an $\mathscr{F}$-morphism (that is, if $S$ is closed in $L$ under the formation of arbitrary infs and finite sups).

3.6. Lemma. Let $S$ be a $\bigwedge$-sublattice of a complete lattice $L$. If $\operatorname{COPRI}(L)$ is a base of $L$, then $\operatorname{COPRI}(S)$ is a base of $S$.

Proof. For $p \in \operatorname{COPRI}(L)$, let $p^{*}=\bigwedge\{x \in S \mid p \leq x\}$. Then $p^{*} \in \operatorname{COPRI}(S)$ and $p \leq p^{*}$. For each $y \in S$, we have $y=\bigvee_{L}\{p \in \operatorname{COPRI}(L) \mid p \leq y\} \leq$ $\bigvee_{S}\left\{p^{*} \mid p \in \operatorname{COPRI}(L)\right.$ and $\left.p \leq y\right\} \leq \bigvee_{S}\{q \in \operatorname{COPRI}(S) \mid q \leq y\}$. It follows that $\operatorname{COPRI}(S)$ is a base of $S$.

For a complete lattice $L$, let $\overline{\mathscr{F}}=\bigcap\{M \subset \mathscr{P}(L) \mid \mathscr{F} \subset M$ and $M$ satisfies $(\dagger \dagger),(* *)\}$. Then $\mathscr{F} \subset \overline{\mathscr{F}}$ and $\overline{\mathscr{F}}$ satisfies $(\bullet \bullet),(\dagger \dagger),(* *)$.

3.7. Theorem. Let $L$ be a complete lattice. Consider the following conditions:

(1) $L$ is strongly $\mathscr{F}$-continuous.

(2) $L$ is weakly $\mathscr{F}$-continuous and $\Downarrow_{\mathscr{F}} x \in I_{\mathscr{F}}(L)$ for all $x \in L$ (that is, $L$ is $\mathscr{F}$-continuous in the sense of [2],[11]).

(3) $L$ is $\mathscr{F}$-continuous.

(4) There is an $I \mathscr{F}^{\dagger}$-embedding of $L$ into a cube $[0,1]^{X}$.

(5) $\operatorname{COPRI}(L)$ is a base of $L$.

(6) $L$ is isomorphic to the lattice of closed sets of a topological space.

(7) $L$ is $\overline{\mathscr{F}}$-distributive.

(8) $L$ is strongly $\overline{\mathscr{F}}$-continuous.

$\left(8^{\prime}\right) L$ is $\overline{\mathscr{F}}$-continuous. 
$\left(8^{\prime \prime}\right) L$ is weakly $\overline{\mathscr{F}}$-continuous.

(9) There is an $\overline{I \mathscr{F}}^{\uparrow}$-embedding of $L$ into a cube $[0,1]^{X}$.

(10) There is a subset system $M$ of $L$ such that

(a) $\mathscr{F} \subset M$ and $M$ satisfies $(\dagger),(*)$, and

(b) $L$ is $M$-distributive.

(11) There is a subset system $M$ of $L$ such that

(a) $\mathscr{F} \subset M$ and $M$ satisfies $(\dagger),(*)$, and

(b) $L$ can be embedded into a cube $[0,1]^{X}$ via an $I M^{\uparrow}$-map.

(12) $L$ is $\mathscr{F}$-distributive.

Then the following implications and equivalences hold:

$$
\begin{aligned}
(1) & \Leftrightarrow(2) \Rightarrow(3) \Rightarrow(4) \Leftrightarrow(5) \Leftrightarrow(6) \Leftrightarrow(7) \Leftrightarrow(8) \Leftrightarrow\left(8^{\prime}\right) \Leftrightarrow\left(8^{\prime \prime}\right) \Leftrightarrow(9) \\
& \Leftrightarrow(10) \Leftrightarrow(11) \Rightarrow(12) .
\end{aligned}
$$

If in L $\mathscr{F}$ satisfies $(\dagger)$, then $(12) \Rightarrow(1)$ is true, and hence all fourteen conditions are equivalent.

Proof. (1) $\Leftrightarrow(2)$. By Lemma 1.8.

(2) $\Rightarrow(3)$. Trivial.

(3) $\Rightarrow(4)$. By Theorem 3.1.

$(4) \Rightarrow(5)$. By Lemma 3.6.

$(5) \Leftrightarrow(6)$. See [12, Proposition 1] (see also [6], [17]).

$(5) \Rightarrow(7)$. Let $M=\{S \subset L \mid$ for each $p \in \operatorname{COPRI}(L), p \leq \bigvee S$ implies $p \leq s$ for some $s \in S\}$. Then $\mathscr{F} \subset M$ and $M$ satisfies $(* *)$. It is easy to check that $L$ is $M$-distributive and $M$ satisfies $(\dagger \dagger)$. Hence $L$ is $\bar{F}$-distributive.

(7) $\Leftrightarrow(8) \Leftrightarrow\left(8^{\prime}\right) \Leftrightarrow\left(8^{\prime \prime}\right) \Leftrightarrow(9)$. By Theorem 3.1.

$(7) \Rightarrow(10)$. Trivial.

$(10) \Leftrightarrow(11)$. By Theorem 3.1.

$(11) \Rightarrow(4)$ and $(10) \Rightarrow(12)$. Trivial.

By Corollary $1.9,(1) \Leftrightarrow(12)$ holds if and only if $\mathscr{F}$ satisfies $(\dagger)$.

However, it still remains open whether the implications (12) $\Rightarrow(3)$ and (12) $\Rightarrow$ (4) always hold.

\section{ACKNOWLEDGMENTS}

Special thanks are due to the National Tianyuan Mathematics Foundation of China for the financial support of this co-operative research with Sichuan University, and to Professor Yingming Liu, the vice-president of Sichuan University, for his interest in this work and constant encouragement.

\section{REFERENCES}

1. H.-J. Bandelt, M-distributive lattices, Arch. Math. (Basel) 39 (1982), 436-442.

2. H.-J. Bandelt and M. Erné, The category of Z-continuous posets, J. Pure Appl. Algebra 30 (1983), 219-226.

3. (1984), 315-324.

4. P. Bernays, A system of axiomatic set theory. III, J. Symbolic Logic 7 (1942), 65-89.

5. G. Bruns, Distributivität und subdirekte Zerlegbarkeit vollständiger Verbände, Arch. Math. (Basel) 12 (1961), 61-66. 
6. D. Drake and W. J. Thron, On the representations of an abstract lattice as the family of closed sets of a topological space, Trans. Amer. Math. Soc. 120 (1965), 57-71.

7. G. Gierz et al., A compendium of continuous lattices, Springer-Verlag, Berlin, 1980.

8. K. H. Hofmann and A. R. Stralka, The algebraic theory of Lawson semilattices-Applications of Galois connections to compact semilattices, Dissertationes Math./Rozprawy Mat. 137 (1976).

9. J. D. Lawson, Topological semilattices, J. London Math. Soc. 2 (1969), 719-724.

10. A. Levy, Basic set theory, Springer-Verlag, Berlin, 1979.

11. D. Novak, Generalization of continuous posets, Trans. Amer. Math. Soc. 272 (1982), 645667.

12. S. Papert, Which distributive lattices are lattices of closed sets?, Proc. Cambridge Philos. Soc. 55 (1959), 172-176.

13. G. N. Raney, A subdirect-union representation for completely distributive complete lattices, Proc. Amer. Math. Soc. 4 (1953), 518-522.

14. _ Tight Galois connections and complete distributivity, Trans. Amer. Math. Soc. 97 (1960), 418-426.

15. D. S. Scott, Outline of a mathematical theory of computation, Proc. 4th Annual Princeton Conf. Information Science and Systems, Princeton Univ. Press, Princeton, NJ, 1970, pp. 169-176.

16. _ Continuous lattices, Toposes, Algebraic Geometry and Logic, Lecture Notes in Math., vol. 274, Springer-Verlag, Berlin, 1972, pp. 97-136.

17. W. J. Thron, Lattice equivalence of topological spaces, Duke Math. J. 29 (1962), 671-680.

Department of Mathematics, Jiangxi Normal University, Nanchang, JiangXi 330027 , People's Republic of China 